\title{
Tolerability and Acceptability of Non-Vitamin K Antagonist Oral Anticoagulants in Atrial Fibrillation: Systematic Review and Meta-Analysis
}

\author{
Daniel Caldeira $^{1,2,3} \cdot$ Nilza Gonçalves $^{1} \cdot$ Joaquim J. Ferreira $^{1,2} \cdot$ Fausto J. Pinto $^{4}$ • \\ João Costa ${ }^{1,2,5,6}$
}

(C) Springer International Publishing Switzerland 2015

\begin{abstract}
Background The non-vitamin $\mathrm{K}$ antagonist oral anticoagulants (NOACs) overcame some limitations of vitamin $\mathrm{K}$ antagonists (VKAs), and are at least as effective in stroke prevention, with an additional decrease of intracranial bleeding risk. The transferability of these benefits to the real world requires tolerability (related to adverse events) and acceptability (drug discontinuation) profiles at least similar to VKAs. Methods We performed a systematic review with metaanalysis of randomized controlled trials (RCTs) evaluating NOACs versus VKAs in patients with non-valvular atrial fibrillation (AF). Studies were searched in April 2015 through MEDLINE, the Cochrane Collaboration's Database, Health Technology Assessment (HTA), Web of
\end{abstract}

Electronic supplementary material The online version of this article (doi:10.1007/s40256-015-0132-5) contains supplementary material, which is available to authorized users.

Daniel Caldeira

dgcaldeira@hotmail.com

1 Clinical Pharmacology Unit, Instituto de Medicina Molecular, Lisbon, Portugal

2 Laboratory of Clinical Pharmacology and Therapeutics, Faculty of Medicine, University of Lisbon, Av. Prof. Egas Moniz, Lisboa 1649-028, Portugal

3 Cardiology Department, Hospital Garcia de Orta, Almada, Portugal

4 Cardiology Department, CCUL, CAML, Faculty of Medicine, Lisbon, Portugal

5 Evidence Based Medicine Centre (CEMBE), Faculty of Medicine, University of Lisbon, Lisbon, Portugal

6 Portuguese Collaborating Centre of the Cochrane Iberoamerican Network, Faculty of Medicine, University of Lisbon, Lisbon, Portugal
Science, and regulatory agencies' documents. Serious adverse events (SAEs) as well as drug-related and patientrelated discontinuation rates were the outcomes of interest. Random-effects meta-analysis was performed, and the results expressed as risk ratios (RRs) and $95 \%$ confidence intervals (CIs). Heterogeneity was evaluated with $I^{2}$ test. Results Five RCTs evaluating four NOACs (apixaban, dabigatran, edoxaban, and rivaroxaban) and 72,720 patients were included. Overall, NOACs were associated with a $4 \%$ risk reduction of SAEs (95\% CI 2-6; $\left.I^{2}=0 \%\right)$. Drug-related and patient-related discontinuation rates were similar between NOACs and VKAs (RR 1.03 [0.88-1.21] and RR 0.99 [0.89-1.10], respectively). Significant heterogeneity $\left(I^{2} \geq 75 \%\right)$ was found among studies results, which could be, at least partially, explained by the findings of the open-label dabigatran trial.

Conclusions NOACs were associated with a small, yet significant, risk reduction of SAEs in patients with AF. NOACs' drug-related and patient-related acceptability profiles were similar to those for VKAs. The results were heterogeneous mainly because of the increased rate of discontinuation associated with dabigatran. Pragmatic trials and cohort studies should be conducted to further address these important clinical questions.

\section{Key Points}

Non-vitamin K antagonist oral anticoagulants (NOACs) reduced significantly the risk of any serious adverse event.

Treatment discontinuation rates were similar between NOACs and warfarin, but there was substantial heterogeneity, mostly related to the RELY trial. 


\section{Introduction}

The non-vitamin $\mathrm{K}$ antagonist oral anticoagulants (NOACs), such as apixaban, dabigatran, edoxaban, and rivaroxaban were recently licensed for the prevention (after hip or knee arthroplasty) and treatment of venous thromboembolism, as well as for stroke and systemic embolism prevention in patients with non-valvular atrial fibrillation (NVAF). While venous thromboembolism treatment or prevention may require only temporary anticoagulant treatment $[1,2]$, stroke prevention in atrial fibrillation (AF) demands life-long treatment. As in any chronic treatment, its effectiveness depends on tolerability and patients' adherence to the medication.

In randomized controlled trials (RCTs), NOACs were at least as effective as vitamin $\mathrm{K}$ antagonists (VKAs) in preventing stroke and systemic embolism, and were associated with a decreased risk of intracranial bleeding [3]. They have overcome many other limitations of VKAs, namely the variability in dose response and the convenience related to absence of frequent coagulation monitoring and dose adjustments.

However, these life-long potential clinical benefits only outweigh the limitations if the adverse reactions and medication adherence profile is at least similar to that experienced by patients treated with VKAs. In the present systematic review, we aimed to evaluate the tolerability and acceptability of NOACs in patients with AF, as these patients require long-term anticoagulation.

\section{Methods}

Preferred Reporting Items for Systematic Reviews and Meta-analyses (PRISMA) framework guidelines were used for reporting guidance [4].

\subsection{Search Strategy}

We searched MEDLINE (Ovid), the Cochrane Collaboration's Database (Ovid), Health Technology Assessment (HTA), and ISI Web of Science, all until April 2015. The search strategy was adapted from the Cochrane Highly Sensitive Search Strategy for identifying randomized trials in humans [5], and is detailed in the supplementary electronic material (see online resource 1). Food and Drug Administration (FDA) and European Medicines Agency (EMA) reports were also consulted for additional unpublished data. Reference lists of retrieved studies and review papers were also cross-checked.

\subsection{Study Selection (Eligibility Criteria) and Data Collection}

We searched for RCTs comparing NOACs with VKAs. We only considered for analysis phase III RCTs because we aimed to determine the risk associated with the approved and commonly used doses of the NOACs and to avoid bias in risk estimation due to the impact of small size underpowered studies on meta-analysis results [6-9]. Furthermore, phase II RCTs have small follow-up periods, which undermines the aim of our review, which is to evaluate the acceptability and tolerability in patients with AF (requiring long-term anticoagulation). Studies where acetylsalicylic acid was used as a single control arm were excluded.

Patients included in studies were required to have a diagnosis of AF with an indication for anticoagulation. Patients with atrial flutter were also included because the procedures in terms of risk stratification and anticoagulation should be the same as in atrial fibrillation. Studies had to report detailed data about serious adverse events (SAEs) and reasons for drug discontinuations. The titles and abstracts of obtained records were screened independently by two authors. Doubts and disagreements were solved by a third person. Selected studies were assessed in full-text to determine their appropriateness for inclusion. Data about study design, patients' characteristics, interventions, and data of required outcomes were retrieved.

Quality of reporting was independently analyzed using the Cochrane Collaboration's Tool [10], which evaluates the following items: random sequence generation method, allocation concealment, blinding of participants and personnel, blinding of outcome assessment, incomplete outcome data, selective reporting, description of withdrawals and other risk of bias features deemed to be important by investigators.

\subsection{Outcome Measures}

The outcomes of interest were tolerability and acceptability of NOACs.

Tolerability was indirectly evaluated by determining the incidence of any SAE, as reported by investigators and/or adjudicated by committees. Whenever possible, treatmentemergent SAEs were retrieved.

Acceptability was split into drug-related (also associated with the tolerability profile) and patient-related treatment discontinuation [11]. Discontinuations due to adverse events were considered to be drug related, and discontinuations due to patients' own decisions (consent withdrawal and treatment discontinuation) were considered to be patient related. Whenever possible, the denominator of these outcomes was the safety population of each arm (i.e., patients that took the drug). 


\subsection{Statistical Analysis}

Statistical analyses were performed using RevMan 5.3 software (The Nordic Cochrane Centre, The Cochrane Collaboration, 2011). Individual studies and meta-analysis estimates were derived and presented in forest plots.

For the meta-analysis, we used the random-effects model weighted by the inverse-variance method to estimate pooled risk ratios (RRs) and $95 \%$ confidence intervals (CIs) [12]. This method was used by default independently of the heterogeneity of the pooled analysis. RRs were chosen to report the results because relative measures tend to be more similar across studies than absolute estimates in populations having different baseline characteristics and lengths of follow-up [13]. Results were evaluated through $Z$ test, and these were considered significant if $p<0.05$.

Heterogeneity, defined as variation beyond chance, was evaluated through the $I^{2}$ test that measures the percentage of total variation between studies [14]. Heterogeneity was considered to be substantial if $I^{2} \geq 50 \%$.

When results were statistically significant, we calculated the number of patients needed to treat (NNT) to expect the avoidance of one event, and the number of events avoided per 1000 treated patients, using as baseline risk the event rate reported in VKA-treated patients $[15,16]$.

Because of the expectation of inclusion of both openlabel and blinded RCTs, and considering the possible influence of these characteristics in the analyzed outcomes, we prespecified a subgroup analysis according to the blinding status of included trials [17]. Despite the distinctive pharmacokinetic and pharmacodynamic properties of individual NOAC drugs, we hypothesized that these drugs could have a class effect compared with VKAs, as shown for some outcomes (e.g., intracranial hemorrhage). Therefore, we did not plan an a priori subgroup analysis considering each individual NOAC drug.

Publication bias was assessed through visual inspection of funnel plots asymmetry. Egger and Peters tests were performed to assess objectively this risk $[18,19]$.

\section{Results}

\subsection{Results of the Search and Description of Studies}

After a comprehensive search for RCTs fulfilling our eligibility criteria, we were able to include five phase III RCTs evaluating four NOACs: apixaban, dabigatran, edoxaban, and rivaroxaban (two studies with rivaroxaban) [20-24].

Supplementary Figure 1 shows the flowchart of study selection, with the reasons for study exclusion (see online resource 1).
Altogether, these trials enrolled 72,720 patients with NVAF under oral anticoagulant treatment, $59 \%$ of them treated with NOACs. Supplementary Table 1 details the main characteristics of included studies.

Overall, the risk of bias was moderate according to the qualitative Cochrane Collaboration Tool (Supplementary Figure 2). We considered that all trials had a high risk of selective reporting because the reporting of any adverse event and its degree is prone to such bias. Additionally the randomized evaluation of long term anticoagulant therapy with dabigatran etexilate (RE-LY) trial had an open-label design [21].

\subsection{Tolerability and Acceptability}

NOACs were associated with a small yet significant $4 \%$ risk reduction of SAEs in patients with NVAF (RR 0.96; $95 \%$ CI 0.94-0.98; Fig. 1a). The results were consistent across studies, without any statistical heterogeneity $\left(I^{2}=0 \%\right)$. NNT with NOACs to expect the prevention of one SAE compared with VKAs was 74 (95 \% CI 49-148) for an average period of 1.7 years. For each 1000 patients treated with NOACs instead of VKAs, it is expected that 14 SAEs (95\% CI 7-20) would be prevented for an average period of 1.7 years.

The drug discontinuation rate due to adverse events was similar between NOACs and VKAs (RR 1.03; $95 \%$ CI 0.88-1.21; Fig. 1b). This analysis was remarkable for significant statistical heterogeneity $\left(I^{2}=93 \%\right)$.

Patient-related drug discontinuation was also similar between NOACs and VKAs (RR 0.99; 95 \% CI 0.89-1.10; Fig. 1c), again showing significant statistical heterogeneity $\left(I^{2}=75 \%\right)$.

\subsection{Subgroup Analysis According to Study Design}

The RE-LY study (dabigatran vs. VKA) was the only openlabel trial [21]. The risk reduction of SAEs was not different between blinded and open-label RCTs ( $p=0.49$; Table 1$)$.

For both drug- and patient-related treatment discontinuations, the results for dabigatran versus VKA (derived from the open-label RE-LY trial) were significantly different compared with the pooled results for the other NOACs ( $p<0.0001$ and $p=0.0001$, respectively). Dabigatran was associated with a significant increase of both drug- and patient-related treatment discontinuations, while pooled results for the other NOACs versus VKAs showed a reduction in the risk of discontinuation due to either adverse events or patients' own decisions (Table 1). The RE-LY trial reported a high number of study discontinuations in dabigatran-treated patients due to gastrointestinal adverse events [21]. The level of heterogeneity in pooled estimates for discontinuation due to drug- and patient-related causes decreased when the RE-LY trial was removed from the analysis $\left(I^{2}=67 \%\right.$ and $I^{2}=0 \%$, respectively). 


\begin{tabular}{|c|c|c|c|c|c|c|}
\hline \multirow{11}{*}{ A } & & \multicolumn{2}{|c|}{ NOACs } & \multicolumn{2}{|c|}{ VKA } & \multirow[b]{2}{*}{ Weight } \\
\hline & Study or Subgroup & Events & Total & Events & Total & \\
\hline & ARISTOTLE & 3182 & 9088 & 3302 & 9052 & $29.4 \%$ \\
\hline & ENGAGE-AF & 5148 & 14014 & 2698 & 7012 & $33.2 \%$ \\
\hline & J-ROCKET & 151 & 639 & 155 & 639 & $1.2 \%$ \\
\hline & RE-LY & 2552 & 12042 & 1357 & 5998 & $13.2 \%$ \\
\hline & ROCKET AF & 2489 & 7111 & 2598 & 7125 & $23.0 \%$ \\
\hline & Total $(95 \% \mathrm{Cl})$ & & 42894 & & 29826 & $100.0 \%$ \\
\hline & Total events & 13522 & & 10110 & & \\
\hline & Heterogeneity: $\left.\right|^{2}=0 \%$ & & & & & \\
\hline & Test for overall effect: & $=4.24($ & $P<0.00$ & & & \\
\hline
\end{tabular}

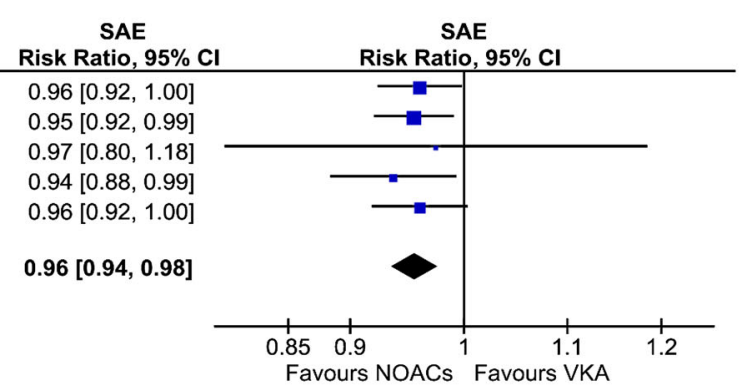

\begin{tabular}{|c|c|c|c|c|c|c|}
\hline \multirow{11}{*}{ B } & \multirow[b]{2}{*}{ Study or Subgroup } & \multicolumn{2}{|c|}{ NOACs } & \multicolumn{2}{|c|}{ VKA } & \multirow[b]{2}{*}{ Weight } \\
\hline & & Events & Total & Events & Total & \\
\hline & ARISTOTLE & 679 & 9088 & 738 & 9052 & $21.2 \%$ \\
\hline & ENGAGE-AF & 2547 & 14014 & 1413 & 7012 & $22.6 \%$ \\
\hline & J-ROCKET & 73 & 639 & 70 & 639 & $12.1 \%$ \\
\hline & RE-LY & 2380 & 12042 & 939 & 5998 & $22.3 \%$ \\
\hline & ROCKET AF & 993 & 7111 & 919 & 7125 & $21.8 \%$ \\
\hline & Total $(95 \% \mathrm{Cl})$ & & 42894 & & 29826 & $100.0 \%$ \\
\hline & Total events & 6672 & & 4079 & & \\
\hline & Heterogeneity: $I^{2}=93 \%$ & & & & & \\
\hline & Test for overall effect: $Z$ & $z=0.41$ & $P=0.68$ & & & \\
\hline C & & NOA & & VKE & & \\
\hline & Study or Subgroup & Events & Total & Events & Total & Weight \\
\hline & ARISTOTLE & 921 & 9088 & 989 & 9052 & $25.3 \%$ \\
\hline & ENGAGE-AF & 1061 & 14014 & 551 & 7012 & $23.9 \%$ \\
\hline & J-ROCKET & 26 & 639 & 35 & 639 & $3.9 \%$ \\
\hline & RE-LY & 914 & 12042 & 375 & 5998 & $22.1 \%$ \\
\hline & ROCKET AF & 805 & 7111 & 838 & 7125 & $24.7 \%$ \\
\hline & Total $(95 \% \mathrm{Cl})$ & & 42894 & & 29826 & $100.0 \%$ \\
\hline & Total events & 3727 & & 2788 & & \\
\hline & Heterogeneity: $\left.\right|^{2}=75 \%$ & & & & & \\
\hline & Test for overall effect: $Z$ & $=0.11$ & $D=001$ & & & \\
\hline
\end{tabular}

Fig. 1 Forest plot with meta-analysis for a SAE risk, b drug-related treatment discontinuation risk, and c patient-related treatment discontinuation risk. ARISTOTLE apixaban for the prevention of stroke in subjects with atrial fibrillation, $C I$ confidence interval, $E N G A G E-A F$ global study to assess the safety and effectiveness of edoxaban vs standard practice of dosing with warfarin in patients with atrial fibrillation, J-ROCKET rivaroxaban versus warfarin in Japanese

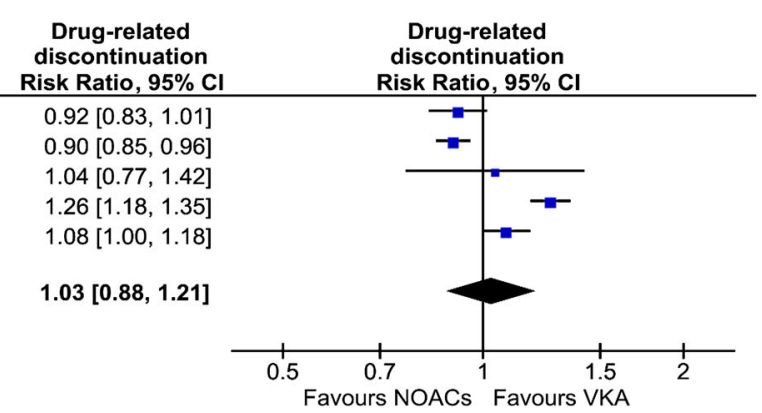

patients with non-valvular atrial fibrillation, NOAC non-vitamin $\mathrm{K}$ antagonist oral anticoagulant, $R E-L Y$ randomized evaluation of long term anticoagulant therapy with dabigatran etexilate, ROCKET-AF an efficacy and safety study of rivaroxaban with warfarin for the prevention of stroke and non-central nervous system systemic embolism in patients with non-valvular atrial fibrillation, $S A E$ serious adverse event, $V K A$ vitamin $\mathrm{K}$ antagonist

Table 1 Results of analysis according to study design

\begin{tabular}{|c|c|c|c|c|c|c|c|c|c|c|}
\hline \multirow[t]{2}{*}{ Trial design } & \multirow[t]{2}{*}{$\mathrm{RCT} /$ patients } & \multicolumn{3}{|l|}{ SAE } & \multicolumn{3}{|c|}{ Drug-related discontinuation } & \multicolumn{3}{|c|}{ Patient-related discontinuation } \\
\hline & & $\begin{array}{l}\mathrm{RR} \\
{[95 \% \mathrm{CI}]}\end{array}$ & $I^{2}$ & $\begin{array}{l}p \text { value for } \\
\text { interaction }\end{array}$ & $\begin{array}{l}\mathrm{RR} \\
{[95 \% \mathrm{CI}]}\end{array}$ & $I^{2}$ & $\begin{array}{l}p \text { value for } \\
\text { interaction }\end{array}$ & $\begin{array}{l}\mathrm{RR} \\
{[95 \% \mathrm{CI}]}\end{array}$ & $I^{2}$ & $\begin{array}{l}p \text { value for } \\
\text { interaction }\end{array}$ \\
\hline $\begin{array}{l}\text { Double-blinded } \\
\text { RCTs }\end{array}$ & $4 / 54,680$ & $\begin{array}{l}0.96 \\
{[0.94-0.98]}\end{array}$ & $0 \%$ & 0.49 & $\begin{array}{l}0.95 \\
{[0.87-1.04]}\end{array}$ & $67 \%$ & $<0.0001$ & $\begin{array}{l}0.95 \\
{[0.90-0.99]}\end{array}$ & $0 \%$ & $<0.0001$ \\
\hline $\begin{array}{l}\text { Open-label } \\
\text { RCT }\end{array}$ & $\begin{array}{c}1 \text { (RE-LY)/ } \\
18,040\end{array}$ & $\begin{array}{l}0.94 \\
\quad[0.88-0.99]\end{array}$ & N/A & & $\begin{array}{l}1.26 \\
\quad[1.18-1.35]\end{array}$ & N/A & & $\begin{array}{l}1.21 \\
\quad[1.08-1.36]\end{array}$ & N/A & \\
\hline
\end{tabular}

$C I$ confidence interval, $R C T$ randomized controlled trial, $R R$ risk ratio, $S A E$ serious adverse event, $N A$ not available

We further performed an exploratory analysis by adding both drug- and patient-related treatment discontinuations into a single outcome. As expected, overall NOACs did reduce the incidence of this outcome (RR 1.00; $95 \%$ CI $0.88-1.14$; Supplementary Figure 3), with high heterogeneity $\left(I^{2}=95 \%\right)$, mostly due to RE-LY (RR 1.25 ; $95 \%$ CI 1.18-1.32). Without RE-LY, NOACs showed a $5 \%$ reduction of drug discontinuation risk (RR $0.95 ; 95 \%$ CI $0.90-1.00 ; I^{2}=57 \%$; Supplementary Figure 3). 


\subsection{Publication Bias}

The scarcity of studies makes funnel plot evaluation less precise for risk of publication bias assessment [25]. Therefore, we only performed Egger and Peters tests, and these were not suggestive for publication bias ( $p \geq 0.25$ for all outcomes and statistical tests).

\section{Discussion}

NOACs (apixaban, dabigatran, edoxaban, and rivaroxaban) are associated with a small, yet clinical significant, decrease in the risk of SAEs when compared with VKAs, without differences between drugs, suggesting a drug-class effect regarding this outcome. However, acceptability, as evaluated through the rate of treatment discontinuation (whether drug related or patient related) was heterogeneous, but not different from VKAs.

The results found among SAEs may be clinically relevant as they reflect differences in the tolerability profile, with lower risk of events with NOACs. Despite the different criteria attributable to the seriousness of an adverse event, among other reasons, these are related to fatal or life-threatening events, disabilities, or situations that require or prolong hospitalization. Therefore, a reduction of events associated with such outcomes may decrease the burden associated with anticoagulated NVAF patients.

Chatterjee et al. [26] have published a review evaluating treatment discontinuations with NOACs. They concluded that NOACs (vs. placebo) had a higher rate of drug discontinuation in patients with acute coronary syndrome. However, NOACs were not significantly different from those with conventional drugs in terms of drug discontinuation in NVAF and venous thromboembolism patients [26]. In our review, different to Chatterjee and colleagues, we considered edoxaban data (ENGAGE AF-TIMI 48: Global study to assess the safety and effectiveness of edoxaban vs standard practice of dosing with warfarin in patients with atrial fibrillation) and excluded studies that could increase bias and decrease precision, such as phase II RCTs and acetylsalicylic acid-controlled trials. Furthermore, we concluded that NOACs decrease the risk of SAEs, which is an important addition to the current knowledge.

It is known that complex therapeutic regimens are important risk factors for non-adherence [27, 28]. It is conceivable that NOACs could improve patients' acceptance of anticoagulants due to their dose-response predictability and not requiring frequent dose adjustments or assessment of hemostasis parameters. Most of the trials had a doubleblinded design, and patients allocated to NOACs were also treated with sham warfarin and INR monitoring. Therefore, the results retrieved from those trials do not account for the potential benefits of NOACs convenience. In terms of drugrelated discontinuation, and with the exception of dabigatran, NOACs showed an acceptability overlapping that of VKAs. In the RE-LY study, patients treated with dabigatran had higher discontinuation rates. The knowledge about the treatment assigned in RE-LY can, at least partially, explain these findings because patients who know that they are being treated with a new active drug may be more prone to discontinue in the setting of an adverse event. However, the discontinuation rate in the dabigatran group due to adverse events was also significantly higher compared with standard anticoagulation in the double-blinded double-dummy efficacy and safety of dabigatran compared to warfarin for 6 month treatment of acute symptomatic venous thromboembolism (RE-COVER) trial that enrolled patients with venous thromboembolism (hazard ratio 1.33; $95 \%$ CI 1.01-1.76; $p=0.05$ ) [29]. Gastrointestinal symptoms, namely dyspepsia, were the main reason for premature dabigatran discontinuation. Other interventions (e.g., taking the drug with meals, $\mathrm{H}_{2}$ antagonists or proton-pump inhibitors) may be needed to mitigate these symptoms in order to improve gastrointestinal tolerability and drug-related acceptability. Our analysis is relevant to establishing the overall tolerability profile of drugs and to generate information/signs about any suspicious adverse events [30-32].

Concerning patient-related discontinuation (acceptability), no differences were found between NOACs and VKAs (RR 0.99; $95 \%$ CI 0.89-1.10), but excluding the RE-LY trial (open-label study) from the analysis resulted in a $5 \%$ significant reduction of patient-related discontinuation risk with NOACs (RR 0.95; $95 \%$ CI 0.90-0.99). The authors do not have an obvious reason for the higher rate of patientrelated discontinuation among dabigatran-treated patients (RR 1.21; $95 \%$ CI 1.08-1.36), as well as for drug-related discontinuation (RR 1.26; $95 \%$ CI 1.18-1.35). It may be hypothesized that the open-label design, by revealing to the patients which treatment they were taking, may have led some patients to choose to maintain their previous standard treatment, but venous thromboembolism data (doubleblinded RCT) does not support it [29]. Other hypotheses can be related to the reporting of a patient's own motifs having (elicited or not) drug-related symptoms.

This is clinically relevant because oral anticoagulant discontinuation (or switch) is associated with a higher risk of thromboembolic events [33].

\subsection{Limitations}

At outcome level, selective reporting bias was our main concern considering the evaluation of SAEs. SAEs are always clinically relevant, but are diverse and may not be related to studied drugs. Some studies reported details on SAEs, while others were more detailed on frequent adverse 
events (not SAEs). These differences did not allow comparing indirectly NOACs' individual SAEs. Furthermore, only ROCKET-AF (An efficacy and safety study of rivaroxaban with warfarin for the prevention of stroke and non-central nervous system systemic embolism in patients with non-valvular atrial fibrillation) provided data about treatment-emergent SAEs, the while other studies only supplied information about overall adverse events.

Heterogeneity of clinical characteristics (e.g., comorbidities that may influence drug dosages, such as renal dysfunction) and interventions (different NOACs, the same NOAC at different dosages, and the possibility of different co-medications) across the various studies should also be considered. The statistical heterogeneity found in some outcomes is a further limitation. Exploring the potential sources of such heterogeneity, we found that dabigatran showed a different acceptability profile to other NOACs. Nevertheless, the reported NOAC results (with or without dabigatran) were consistent in terms of direction and significance of estimates (acceptability profile similar to that for VKAs).

Finally, we accessed tolerability and acceptability on the basis of data from exploratory clinical trials in a tightly controlled environment. These outcomes are better evaluated in pragmatic trials and from "real-world" data.

\section{Conclusions}

Overall, NOACs are associated with a small, yet potentially clinically significant, $4 \%$ reduction in the risk of SAEs. NOACs' drug-related and patient-related acceptability profiles were similar to those for VKAs. At this level, NOACs did not show a clear drug-class effect. The results were heterogeneous mainly because of the increased rate of discontinuation in dabigatran-treated patients (predominantly associated with gastrointestinal symptoms). These conclusions are driven from randomized data, which is not the most powerful design to evaluate safety, tolerability, and acceptability outcomes. Pragmatic trials and large prospective cohort studies should be conducted to address these important clinical questions.

\section{Acknowledgments Portuguese Collaborating Centre of the Cochrane Iberoamerican Network.}

Contributors DC contributed to the concept and design, data acquisition, data analysis, and interpretation of the data; wrote the first draft of the manuscript; critically revised the manuscript; and gave final approval of the submitted manuscript. JC contributed to the data acquisition and data analysis; critically revised the manuscript; and gave final approval of the submitted manuscript. NG, FJP, and JJF contributed to the interpretation of data, critically revised the manuscript, and gave final approval of the submitted manuscript.
Funding This was an academic project not funded by any government or non-government grants.

Ethical approval Not required.

Competing interests We declare the following potential conflicts of interests: JJF had speaker and consultant fees with GlaxoSmithKline, Novartis, TEVA, Lundbeck, Solvay, Abbott, Bial, Merck-Serono, Grunenthal, and Merck Sharp and Dohme; FJP had consultant and speaker fees with Astra Zeneca, Bayer and Boehringer Ingelheim; the remaining authors do not have any competing interests to disclose.

\section{References}

1. Falck-Ytter Y, Francis CW, Johanson NA, Curley C, Dahl OE, Schulman S, Ortel TL, Pauker SG, Colwell CW Jr, American College of Chest Physicians. Prevention of VTE in orthopedic surgery patients: antithrombotic therapy and prevention of thrombosis, 9th ed: American College of Chest Physicians Evidence-Based Clinical Practice Guidelines. Chest. 2012;141:e278S-325S.

2. Kearon C, Akl EA, Comerota AJ, Prandoni P, Bounameaux H, Goldhaber SZ, Nelson ME, Wells PS, Gould MK, Dentali F, Crowther M, Kahn SR, American College of Chest Physicians. Antithrombotic therapy for VTE disease: antithrombotic therapy and prevention of thrombosis, 9th ed: American College of Chest Physicians Evidence-Based Clinical Practice Guidelines. Chest. 2012;141:e419S-94S.

3. Caldeira D, Barra M, Pinto FJ, Ferreira JJ, Costa J. Intracranial hemorrhage risk with the new oral anticoagulants: a systematic review and meta-analysis. J Neurol 2015;262:516-22. doi:10. 1007/s00415-014-7462-0.

4. Liberati A, Altman DG, Tetzlaff J, Mulrow C, Gøtzsche PC, Ioannidis JP, Clarke M, Devereaux PJ, Kleijnen J, Moher D. The PRISMA statement for reporting systematic reviews and metaanalyses of studies that evaluate health care interventions: explanation and elaboration. PLoS Med. 2009;21(6):e1000100.

5. Lefebvre C, Manheimer E, Glanville J. Chapter 6: Searching for studies. In: Higgins JPT, Green S, editors. Cochrane Handbook for Systematic Reviews of Interventions Version 5.1.0 (updated March 2011). The Cochrane Collaboration, 2011. Available from http://www.cochrane-handbook.org. Accessed Apr 2015.

6. Turner RM, Bird SM, Higgins JP. The impact of study size on meta-analyses: examination of underpowered studies in Cochrane reviews. PLoS One. 2013;8:e59202.

7. Kjaergard LL, Villumsen J, Gluud C. Reported methodologic quality and discrepancies between large and small randomized trials in meta-analyses. Ann Intern Med. 2001;135:982-9.

8. Zhang Z, Xu X, Ni H. Small studies may overestimate the effect sizes in critical care meta-analyses: a meta-epidemiological study. Crit Care. 2013;17:R2.

9. Caldeira D, Barra M, Santos AT, de Abreu D, Pinto FJ, Ferreira JJ, Costa J. Risk of drug-induced liver injury with the new oral anticoagulants: systematic review and meta-analysis. Heart. 2014;100:550-6.

10. Higgins JPT, Altman DG, Sterne JAC. Chapter 8: Assessing risk of bias in included studies. In: Higgins JPT, Green S, editors. Cochrane Handbook for Systematic Reviews of Interventions Version 5.1.0 (updated March 2011). The Cochrane Collaboration, 
2011. Available from http://www.cochrane-handbook.org. Accessed Apr 2015.

11. Cipriani A, Furukawa TA, Salanti G, Geddes JR, Higgins JP, Churchill R, Watanabe N, Nakagawa A, Omori IM, McGuire H, Tansella M, Barbui C. Comparative efficacy and acceptability of 12 new-generation antidepressants: a multiple-treatments metaanalysis. Lancet. 2009;373:746-58.

12. DerSimonian R, Laird N. Meta-analysis in clinical trials. Control Clin Trials. 1986;7:177-88.

13. Deeks JJ. Issues in the selection of a summary statistic for metaanalysis of clinical trials with binary outcomes. Stat Med. 2002;21:1575-600.

14. Deeks JJ, Altman DG, Bradburn MJ. Statistical methods for examining heterogeneity and combining results from several studies in metaanalysis. In: Egger M, Davey Smith G, Altman DG, editors. Systematic reviews in health care: meta-analysis in context, 2nd ed. London: BMJ Publication Group; 2001, p. $313-335$.

15. Walter SD. Number needed to treat $(\mathrm{NNT})$ : estimation of a measure of clinical benefit. Stat Med. 2001;20:3947-62.

16. Schünemann HJ, Oxman AD, Vist GE, Higgins JPT, Deeks JJ, Glasziou P, Guyatt GH. Chapter 12: Interpreting results and drawing conclusions. In: Higgins JPT, Green S, editors. Cochrane Handbook for Systematic Reviews of Interventions Version 5.1.0 (updated March 2011). The Cochrane Collaboration, 2011. Available from http://www.cochrane-handbook.org. Accessed Apr 2015.

17. Beyer-Westendorf $\mathrm{J}$, Büller $\mathrm{H}$. External and internal validity of open label or double-blind trials in oral anticoagulation: better, worse or just different? J Thromb Haemost. 2011;9:2153-8.

18. Egger M, Davey Smith G, Schneider M, Minder C. Bias in metaanalysis detected by a simple, graphical test. BMJ. 1997;315:629-34.

19. Peters JL, Sutton AJ, Jones DR, Abrams KR, Rushton L. Comparison of two methods to detect publication bias in meta-analysis. JAMA. 2006;295:676-80.

20. Granger CB, Alexander JH, McMurray JJ, et al. Apixaban versus warfarin in patients with atrial fibrillation. $\mathrm{N}$ Engl $\mathrm{J}$ Med. 2011;365:981-92.

21. Connolly SJ, Ezekowitz MD, Yusuf S, et al. Dabigatran versus warfarin in patients with atrial fibrillation. $\mathrm{N}$ Engl $\mathrm{J}$ Med. 2009;361:1139-51.

22. Giugliano RP, Ruff CT, Braunwald E, et al. Edoxaban versus warfarin in patients with atrial fibrillation. $\mathrm{N}$ Engl $\mathrm{J}$ Med. 2013;369:2093-104.
23. Patel MR, Mahaffey KW, Garg J, et al. Rivaroxaban versus warfarin in nonvalvular atrial fibrillation. $\mathrm{N}$ Engl $\mathrm{J}$ Med. 2011;365:883-91.

24. Hori M, Matsumoto M, Tanahashi N, et al. Rivaroxaban vs. warfarin in Japanese patients with atrial fibrillation-the J-ROCKET AF study. Circ J. 2012;76:2104-11.

25. Sterne JAC, Egger M, Moher D, editors. Chapter 10: Addressing reporting biases. In: Higgins JPT, Green S, editors. Cochrane Handbook for Systematic Reviews of Intervention. Version 5.1.0 (updated March 2011). The Cochrane Collaboration, 2011. Available from http://www.cochrane-handbook.org. Accessed Apr 2015.

26. Chatterjee S, Sardar P, Giri JS, Ghosh J, Mukherjee D. Treatment discontinuations with new oral agents for long-term anticoagulation: insights from a meta-analysis of 18 randomized trials including 101,801 patients. Mayo Clin Proc. 2014;89:896-907.

27. Caldeira D, Vaz-Carneiro A, Costa J. The impact of dosing frequency on medication adherence in chronic cardiovascular disease: systematic review and meta-analysis. Rev Port Cardiol. 2014;33:431-7.

28. Coleman CI, Limone B, Sobieraj DM, Lee S, Roberts MS, Kaur $\mathrm{R}$, Alam T. Dosing frequency and medication adherence in chronic disease. J Manag Care Pharm. 2012;18:527-39.

29. Schulman S, Kearon C, Kakkar AK, Mismetti P, Schellong S, Eriksson H, Baanstra D, Schnee J, Goldhaber SZ, RE-COVER Study Group. Dabigatran versus warfarin in the treatment of acute venous thromboembolism. $\mathrm{N}$ Engl $\mathrm{J}$ Med. 2009;61:2342-52.

30. Caldeira D, Costa J, Pinto FJ, Ferreira JJ. The risk of infection with new oral anticoagulants: a meta-analysis. Int $\mathrm{J}$ Cardiol. 2014;172:267-8.

31. Caldeira D, Barra M, Gonçalves N, Pinto FJ, Ferreira JJ, Costa J. Pericardial bleeding risk with non-vitamin $\mathrm{K}$ oral anticoagulants: a meta-analysis. Int J Cardiol. 2014;182C:187-8.

32. Caldeira D, Barra M, Santos AT, de Abreu D, Costa J, Ferreira JJ. Risk of insomnia with non-vitamin $\mathrm{K}$ oral anticoagulants: systematic review and meta-analysis. Sleep Breath. 2015. doi:10. 1007/s11325-014-1112-8.

33. Caldeira D, Costa J, Ferreira JJ, Pinto FJ. Thromboembolic risk in the initiation, switch and interruption/re-initiation of oral anticoagulants: do newcomers improve outcomes? Insights from a meta-analysis of RCTs. Int J Cardiol. 2014;177:117-9. 